\title{
Household Food Security Status and Child Health Outcomes in Kenya
}

\author{
Michelle M. Mwangi (MA) \\ Mercy G. Mugo (PhD)
}

School of Economics, University of Nairobi, Kenya

\begin{abstract}
Interminable access to sufficient, nutritious, and safely prepared food is a human right. Attributed to insufficient food and nutrient intake, malnutrition is a major health burden in developing economies that has maimed socioeconomic development. In children, undernourishment impairs the functioning of the immune system, increases susceptibility to diseases, and undermines physical and cognitive development. In Kenya, there exists a paucity of empirical corroboration of the effect of household food security status (HFSS) on child health outcomes. Using data drawn from the 2014 Kenya Demographic and Health Survey, this paper focuses on analyzing the causal link between HFSS and child health outcomes and to provide evidencebased policy recommendations to promote child health outcomes. We employed three measures of HFSS: households that lacked food/enough money to purchase food, the Reduced Coping Strategy Index (CSI), and the Food Consumption Score (FCS). The child health production function was estimated using the two-stage residual inclusion (2SRI) technique to control for potential endogeneity. The results indicate that households that lacked food/enough money to purchase food were significantly associated with stunted, wasted, and underweight growth in children. Similarly, the Reduced CSI was a significant determinant of stunted and underweight growth in children. However, the effect was insignificant relative to wasted growth. The findings also indicate that FCS contributes significantly to improvements in child health outcomes. Our evidence has the potential to inform policies on the promotion of child health outcomes. We recommend implementation of programs such as social assistance, integration of nutrition and WASH, and capacity-building to promote women's knowledge of health, nutrition, and better child-care practices.
\end{abstract}

Keywords: Food security, Child health outcomes, Kenya, IV 2SRI 


\section{Introduction}

Building on the gains of the Millennium Development Goals (MDGs), the United Nations launched the Sustainable Development Goals (SDGs) in 2015 that provided a guideline to steer developed and developing economies in achieving sustainable growth and development by 2030 (United Nations, 2015). Under the SDGs framework, zero hunger and good health and wellbeing goals are interlinked, and it emphasizes the role of food security and good health as prerequisites for socioeconomic development. Food security is a multi-dimensional phenomenon which is defined as the state in which everyone in a given economy has an interminable physical, social, and economic access to food that is adequate, safely prepared, and full of nourishment to meet dietary requirements (Food and Agriculture Organization [FAO], 2009). According to the Global Food Security Index 2018 report that assessed national food security across 113 countries, Singapore is the most food-secure with a score of 85.9 out of 100, followed by Ireland with a score of 85.5 (The Economist Intelligence Unit [EIU], 2018). Kenya, however, is ranked $87^{\text {th }}$ with a score of 41.9 and has experienced a decline in the net food security score by 0.3 between 2017 and 2018 (EIU, 2017, 2018).

Health is an integral aspect of enhancing sustainable growth and development. It contributes to economic growth through five mechanisms: labor productivity, education, savings, resources, and demography (World Bank, 1993; Bloom and Canning, 2008). Food security affects health through non-nutritional and nutritional pathways. On one hand, the non-nutritional pathway relates to the anxiety and uncertainty households experience about their capability to provide adequate and nutritious food. The effects are evident through stress and depression which undermines labor productivity. On the other hand, the nutritional pathway relates to the health effects attached to the varying behavioral strategies households adapt as the severity of food insecurity exacerbates. Some of these coping strategies include consuming cheaper and less nutritious food that compromise the minimum requirements for macro and micronutrients (Meerman and Aphane, 2012). In dire circumstances, households forgo basic needs like medical care to provide food with ripple effects on health. Eventually, deteriorated health status increases out-of-pocket spending on health care which constrains available resources to be allocated to other productive ventures like savings and investments (World Bank, 1993). Moreover, poor health outcomes in children reduce the rate of school enrollment, comprehension, and participation causing a decrease in expected returns to investments in schooling and future labor market outcomes (Bloom and Canning, 2008).

Intertwined with poverty, food insecurity is an underlying determinant of malnutrition with children who are particularly vulnerable to the effects due to the high nutritional requirements essential for growth and development 
(Blössner and de Onis, 2005). According to the Kenya National Bureau of Statistics (KNBS, 2018), 35.8\% of children aged between 0 and 17 years in Kenya are considered food poor with most of this proportion residing in rural areas like Turkana, Samburu, Mandera, and Busia. During the critical stages of growth, severe malnutrition reduces the functioning of the immune system, increases susceptibility to diseases, and impairs physical and cognitive development with effects propagated to future generations. In Kenya, out of the aggregate number of children under-five years, $26 \%, 4 \%$, and $11 \%$ are stunted, wasted, and underweight. This is a contributing factor to under-five mortality rate standing at 52 deaths per 1000 live births (Kenya National Bureau of Statistics [KNBS], Ministry of Health/Kenya, National AIDS Control Council/Kenya, Kenya Medical Research Institute, National Council for Population and Development/Kenya and ICF International, 2015). This is above the set targets under the "good health and well-being" goal to reduce under-five mortality rate to as low as 25 deaths per 1000 live births (United Nations Development Programme [UNDP], 2019).

Globally, researchers have widely recognized the need to study the causal link between household food security status (HFSS) and nutritional outcomes in children. However, studies exploring this relationship have provided mixed results. Some studies show that HFSS is significantly associated with stunting (Hackett, Melgar-Quiñonez and Álvarez, 2009; Jemal, Hassen and Wakayo, 2016; Mutisya, Kandala, Ngware and Kabiru, 2015; Saaka and Osman, 2013; Saha, Frongillo, Alam, Arifeen, Persson and Rasmussen, 2009; Singh, Singh and Ram, 2014), wasting (Kac, Schlüssel, Pérez-Escamilla, Velásquez-Melendez and Moura da Silva, 2012; Saha et al., 2009), and underweight (Hackett et al., 2009; Saha et al., 2009; Singh et al., 2014). Nevertheless, a few studies have discerned no significant association between HFSS and stunting (Osei, Pandey, Spiro, Nielson, Shrestha, Talukder, Quinn and Haselow, 2010), wasting (Jemal et al., 2016; Singh et al., 2014), and underweight (Jemal et al., 2016; Osei et al., 2010).

The discrepancies in existing literature allude to the fact that food security status is a necessary but insufficient determinant of nutritional outcomes. Besides food, factors like intra-household allocation and utilization of food, knowledge of nutritional requirements, and water and sanitation for health (WASH) conditions also determine nutritional outcomes (Jemal et al., 2016). More so, the existence of heterogeneity in literature is attributed to the different methodologies in measuring food security, the distinct contextspecific populations studied, and the varying velocities and stages of nutrition transition (Kac et al., 2012; Saaka and Osman, 2013).

Furthermore, the role that food security plays on nutrition and health outcomes has not been adequately studied especially in developing economies (Saha et al., 2009; Saaka \& Osman, 2013; Jemal et al., 2016). There exists a 
dearth of empirical evidence based on cross-sectional data in analyzing this causal link in Kenya. This study, therefore, seeks to estimate the effect of HFSS on child health outcomes in Kenya using the 2014 KDHS. Specifically, we analyze the effect of HFSS on child health outcomes and suggest policy recommendations to promote child health in Kenya.

\section{Methodology}

\section{Theoretical Framework}

Our study finds its theoretical basis in the health production theory, specifically the household production theory that gained prominence in Gary Becker's work in 1965 on the theory of allocation of time. It provides a unitary framework for analyzing households considered both as consuming and producing units. Huffman (2010) provided an application of this theory to the production of health with food, leisure time, and medical care as key inputs. The production function to be estimated is a modified specification of Huffman's model, given as;

Where;

$$
H=f(H F S S ; W)
$$

- $\quad \mathrm{H}$ is the measure of child nutritional outcomes (stunted, wasted, and underweight) as a proxy of child health status

- HFSS is the state of household food security

- $\mathrm{W}$ is an array of covariates (nutritional knowledge, water and sanitation)

Food is a necessary but insufficient determinant of child nutritional outcomes. This is because improvements in nutrition are not only attributed to the quantity of food intake, but also the quality. Maternal capability to provide quality and balanced diets is determined by their knowledge on nutritional requirements embedded in socioeconomic profiles. Beyond the provision of adequate and nutritious food, aspects of water and sanitation determine the extent of assimilation of essential nutrients.

\section{Estimation Model}

A logistic regression model was estimated to analyze the effect of HFSS on child health outcomes in Kenya. The structural child health production function is given as:

$$
\left.H_{i} \text { (stunted, wasted, underweight }\right)=\beta_{0}+\beta_{1} H F S S_{i}+\beta_{2 j} W_{j}+\varepsilon_{i}
$$

Endogeneity is a major econometric issue present within the health production function, and it is attributed to measurement errors, omitted variables, simultaneity, and reverse causality (Bascle, 2008). Rose and Bodor (2006) stated that food security status may be highly correlated with the disturbance term leading to biased estimates. This is because of the presence of simultaneity between nutritional outcomes and HFSS. Improvements in 
nutritional outcomes stimulate labor productivity and increased income, which in turn enhances HFSS. On the other hand, HFSS affects the consumption of adequate, safe, and nutritious food which determines the extent of improvements in nutritional outcomes.

Instrumental Variable (IV) technique is the most applicable tool in econometrics that allows estimation of consistent structural parameters in the presence of endogeneity (Shea, 1997). The advantage of using IV is that it allows the researcher to partition the variation of the endogenous predictor into exogenous and endogenous components (Bound, Jaeger and Baker, 1995). In non-linear models, IV can be applied through two approaches: Two-Stage Residual Inclusion (2SRI) and Two-stage Predictor Substitution (2SPS). However, Terza, Basu and Rathouz (2008) have proven that 2SPS is inconsistent and therefore recommends the use of 2SRI to control for endogeneity in non-linear models.

2SRI-IV is conducted through two steps and it relies on the identification of instruments for HFSS. For 2SRI-IV estimates to be consistent and suitable for statistical inference, the instruments should be relevant, strong, and exogenous. An instrument that meets all these conditions is said to be valid. However, a major challenge faced by researchers is the identification of valid instruments (Stock, Wright and Yogo, 2002). In the first stage, HFSS is regressed on all exogenous predictors in the model, including its instruments $\left(\mathrm{Z}_{\mathrm{k}}\right)$ and the residual is predicted as;

$$
\text { HFSS }_{i}=\alpha_{0}+\alpha_{1 j} W_{j}+\alpha_{2 k} Z_{k}+\mu_{i}
$$

This study used three measures of HFSS to capture the entire spectrum of the multi-faceted concept; households that lacked food/enough money to purchase food, the Reduced Coping Strategy Index (CSI), and the Food Consumption Score (FCS). Wealth index and land ownership were used as instrumental variables for the households that lacked food/enough money to purchase food and the FCS. On the other hand, wealth index and the number of children under-five years in the household were used as instruments for the Reduced CSI. Unlike linear models, there are no standardized econometric tests to determine the validity of instrumental variables in non-linear models. Therefore, this study used the reduced form estimates to evaluate the validity of the instruments. The second step is to include the predicted residual in equation 2;

$$
H_{i}=\beta_{0}+\beta_{1} H_{F S S_{i}}+\beta_{2 j} W_{j}+\beta_{3} \widehat{H F S S}_{\imath}+\varepsilon_{i}
$$

Based on the Durbin-Wu-Hausman test statistic, if $\beta_{3}$ is significantly different from zero, endogeneity exists within the structural equation. Hence, equation 4 is estimated. However, if $\beta_{3}$ is insignificant, endogeneity is absent and the model to be estimated is given in equation 2 . 


\section{Variable Description}

Nutritional status which was determined using anthropometric z-scores was used to proxy child health outcomes (Table 1).

Table 1. Child Nutritional Status (Dependent variable)

\begin{tabular}{llll}
\hline \multirow{2}{*}{ Malnutrition categories } & \multicolumn{4}{l}{ Nutritional status of children under the age of five years } \\
\cline { 2 - 4 } & Stunted growth & Wasted growth & Underweight growth \\
\hline Normal (=0) & HFAZ $\geq-2$ & WFHZ $\geq-2$ & WFAZ $\geq-2$ \\
Severe $(=1)$ & HFAZ<-2 & WFHZ $<-2$ & WFAZ<-2 \\
\hline
\end{tabular}

Source: WHO (2006)

HFSS is measured using the households that lacked food/enough money to purchase food within seven days preceding the survey. It is a binary variable coded as 1 "Yes" and 0 "No". The second measure is the Reduced CSI determined from follow-up questions based on "Yes" responses to the previous question. Specifically, households that lacked food/enough money to purchase food are then asked to indicate the number of days in a week they relied on various coping strategies. The Reduced CSI is a composite calculation of the frequency of adapting coping strategy $i$, and the severity weight attached to each coping strategy.

$$
\text { Reduced CSI }=\sum f\left(C S_{i}\right) * S W_{i}
$$

Table 2. Weighted Coping Strategies

\begin{tabular}{ll}
\hline Coping strategy (CS) & Severity weight (SW) \\
\hline Rely on less preferred/less expensive food & 1.0 \\
Rely on borrowed food from friends or relatives & 2.0 \\
Reduced the number of meals consumed in a day & 1.0 \\
Reduced the portion size of meals & 1.0 \\
Reduced quantities for adults to provide for the children & 3.0 \\
\hline
\end{tabular}

Source: KNBS et al., 2015; Maxwell and Caldwell, 2008

The last measure of HFSS is the Food Consumption Score (FCS), a composite calculation of consuming food group $i$, and the nutritional value attached to each food group.

$$
F C S=\sum f\left(F G_{i}\right) * N V_{i}
$$

Table 3. Food groups and weighted nutritional value

\begin{tabular}{ll}
\hline Food group (FG) & Nutritional value (NV) \\
\hline Staples & 2 \\
Pulses & 3 \\
Vegetables & 1 \\
Fruits & 1 \\
Meat \& Fish & 4 \\
Milk & 4 \\
Sugar & 0.5 \\
Oil & 0.5 \\
Condiments & 0 \\
\hline
\end{tabular}

Source: WFP, VAM (2006) 
Health and nutrition knowledge among primary caregivers is an essential aspect of improving the quality of child-care practices and health. However, information on women's nutritional knowledge was not available in KDHS (2014). Hence, maternal education was used as a proxy. Source of drinking water was categorized as a binary variable indicating "Improved $=1$ " and "Unimproved=0". Improved water sources include bottled water, protected well, spring water, public tap, standpipe, piped water into dwelling, yard or plot, rainwater, and tube well or borehole. On the contrary, unimproved sources include surface water (river, lake, pond, and dam), tanker truck water, unprotected well, spring water and others (KNBS et al., 2015). The type of sanitation facility was similarly categorized as improved and unimproved. Improved sanitation facilities include composting sanitation, pit latrines with ventilation or slab, flush to sewer, pit latrine or septic tank. Unimproved facilities include bucket and hanging toilets, pit latrines without ventilation or slab, no flush system, no facility or bush and others (KNBS et al., 2015). To control for unobservable characteristics in the child health production function, Table 4 illustrates the various covariates that will be included in the structural model.

Table 4. Variable description

\begin{tabular}{|c|c|}
\hline Variable & Description \\
\hline \multicolumn{2}{|l|}{ Dependent variables } \\
\hline Stunted growth & Categorical variable: 1 "Stunted", 0 "Normal" \\
\hline Wasted growth & Categorical variable: 1 "Wasted", 0 "Normal" \\
\hline Underweight growth & Categorical variable: 1 "Underweight", 0 "Normal" \\
\hline \multicolumn{2}{|c|}{ Household food security status (HFSS)-(Independent variable of interest) } \\
\hline $\begin{array}{l}\text { Households that lacked food/enough money to } \\
\text { purchase food }\end{array}$ & Categorical variable: 1 "Yes", 0 "No" \\
\hline Reduced CSI & Continuous variable \\
\hline FCS & Continuous variable \\
\hline \multicolumn{2}{|l|}{ Covariates } \\
\hline Child's gender & Categorical variable: 1 "Male", 0 "Female" \\
\hline Child's age (months) & Continuous variable \\
\hline Maternal age (years) & Continuous variable \\
\hline Maternal education level & $\begin{array}{l}\text { Categorical variable: } 1 \text { "None", } 2 \text { "Primary", } 3 \\
\text { "Secondary", } 4 \text { "Tertiary" }\end{array}$ \\
\hline Maternal working status & Categorical variable: 1 "Yes", 0 "No" \\
\hline Area of residence & Categorical variable: 1 "Rural', 0 "Urban" \\
\hline Source of drinking water & Categorical variable: 1 "Improved", 0 "Unimproved" \\
\hline Type of sanitation facility & Categorical variable: 1 "Improved", 0 "Unimproved" \\
\hline
\end{tabular}

\section{Data Source}

This study used data from the Kenya Demographic and Health Survey (KDHS, 2014). The sample was drawn from the master sampling frame (NASSEP V) using a two-stage sampling procedure. In the first stage, 1612 clusters were drawn from the master frame with 995 and 617 clusters from 
rural and urban households, respectively. In the second stage, 25 households were randomly selected from each of the clusters. The survey collection was centered around issues on child and maternal health, nutritional status, fertility, mortality, fistula, HIV/AIDS, and violence.

Data collected from the survey was disaggregated into different modules that captured household, men, women, couples, children and birth data. The household data provides information on household characteristics while the children's data provides information on health indicators of children underfive years, as well as their mothers. Data used for this study was obtained by merging the household and the children's datasets, using cluster and household number as unique identifiers. The inclusion criterion for this analysis is children under-five years.

\section{Results}

\section{Sample Description}

Merging the household and children data modules linked 20,964 individual household characteristics to the health status of a child living within the household. Out of these, only 18,656 observations were included in the sample as 2,308 physiologically implausible flagged cases and missing anthropometric indices were excluded from the analysis.

Table 5 presents mean and standard deviations for the sample. The findings show that $27 \%, 5 \%$, and $13 \%$ of children under-five years were stunted, wasted, and underweight, respectively. The average number of children under-five years living within the household was nearly 2 (mean=1.84, $\mathrm{SD}=0.85)$. Most of the children were male $(51 \%)$, with an average age of 29.11 months $(\mathrm{SD}=16.98)$. More than half of the mothers had attained primary level of education (53\%) and were currently working (58\%), with a mean age of 28.81 years $(\mathrm{SD}=6.55)$.

More than half of the sample consisted of poor households (56\%), residing in rural areas (69\%). Most of the households owned land (64\%). This gives a plausible explanation as to why fewer (37\%) households lacked food or enough money to purchase food within seven days preceding the survey. The average Reduced CSI and FCS was 19.21 (IQR: 9-28) and 85.94 (IQR: 60-112), respectively. Although most of the households had improved sources of drinking water $(60 \%)$, only two out of five had improved sanitation facilities $(41 \%)$. 
Table 5. Sample Descriptive Statistics

\begin{tabular}{lcc}
\hline Variable & $\mathbf{n}$ & Mean (SD) \\
\hline Dependent variables & \multicolumn{1}{l}{} \\
Stunted growth & 18,656 & $0.27(0.44)$ \\
Wasted growth & 18,656 & $0.05(0.23)$ \\
Underweight growth & 18,656 & $0.13(0.34)$ \\
Household food security status (HFSS) - (Independent variable of interest) \\
Households that lacked food/enough money to purchase & 9,028 & $0.37(0.48)$ \\
food & & \\
Reduced CSI & 3,385 & $19.21(13.01)$ \\
& & IQR: $[9-28]$ \\
FCS & 18,656 & $85.94(30.25)$ \\
& & IQR: $[60-112]$ \\
Covariates & & \\
Child's gender (1=Male) & 18,656 & $0.51(0.49)$ \\
Child's (months) & 18,656 & $29.11(16.98)$ \\
Maternal age (years) & 18,656 & $28.81(6.55)$ \\
Maternal education level & & \\
(1=None) & 18,656 & $0.22(0.41)$ \\
(1=Primary) & 18,656 & $0.53(0.49)$ \\
(1=Secondary) & 18,656 & $0.19(0.39)$ \\
(1=Tertiary) & & $0.06(0.24)$ \\
Maternal working status (1=Yes) & 9,027 & $0.58(0.49)$ \\
Area of residence (1=Rural) & 18,656 & $0.69(0.46)$ \\
Source of drinking water (1=Improved) & 18,649 & $0.60(0.49)$ \\
Type of sanitation facility (1=Improved) & 18,651 & $0.41(0.49)$ \\
Instrumental variables & & \\
Wealth Index & & \\
(1=Poor) & 18,656 & $0.56(0.49)$ \\
(1=Middle) & 18,656 & $0.17(0.37)$ \\
(1=Rich) & 18,656 & $0.27(0.45)$ \\
Ownership of land (1=Yes) & 18,653 & $0.64(0.48)$ \\
Number of children in the household under-five years & 18,656 & $1.84(0.85)$ \\
\hline & &
\end{tabular}

Source: Author's computation from KDHS (2014)

\section{Instrumenting Household Food Security Status}

Tables 6,7, and 8 present regression results on the three measures of HFSS and child health outcomes. From the findings (Tables 6 and 8), the significance of the residuals is an indication of the presence of endogeneity within the models. This provides a justification for the use of the 2SRI-IV estimation technique. Table 7 shows that endogeneity was not present in the wasted growth model as indicated by the insignificance of the residual Reduced CSI. Hence, the model was estimated using the standard logistic regression approach.

The results from the reduced form estimates in Table 6 and 8 indicate that wealth index and land ownership were significantly associated with the households that lacked food or enough money to purchase food and the FCS. This indicates that the instruments were valid. Households that belong to the 
wealthiest quintile and owned land were less likely to lack food/enough money to purchase food as compared to those among the poorest quintiles that did not own any parcels of land. In relation to the FCS, households ranked among the highest wealth quintiles and owned land were significantly associated with an increase in the FCS. With every unit increase in the FCS, households are predisposed to the consumption of acceptable food groups.

In Table 7, wealth index and the number of children under-five years within a household were significant predictors of the Reduced CSI. Wealthier households can provide adequate and nutritious food. Hence, there is a decrease in the Reduced CSI. One of the coping strategies households adapt in the wake of food insecurity is to reduce the food intake for adults to provide for the children. Therefore, as the number of children under-five years within a household increases, the Reduced CSI increases.

\section{Impact of Household Food Security Status on Child Health Outcomes in Kenya}

Table 6 presents regression results on the impact of lack of food or enough money to buy food on health status in terms of odds ratio of experiencing stunting, wasting, and underweight. Results show that children born into households that lacked food or enough money to purchase food were significantly more likely $(94 \%)$ to experience stunting, and two times more likely to suffer from wasted $(\mathrm{OR}=2.991)$ and underweight growth $(\mathrm{OR}=2.890)$. For every unit increase in the Reduced CSI, children under-five years were $7.6 \%$ and $1.6 \%$ more likely to suffer from stunted and underweight growth, respectively. Although there was a slight increase in the likelihood of a child suffering from wasted growth for every unit increase in the Reduced CSI, the results were insignificant. This supports similar findings (Jemal et al., 2016; Singh et al., 2014) where no significant association was established between HFSS and wasted growth. This is an indication that nutritional outcomes in children are a complex phenomenon that is not only determined by the provision of adequate and nutritious food but also access to health care, nutritional knowledge, intra-household allocation of food, and WASH conditions. On the contrary, for every unit increase in the FCS, the likelihood of a child suffering from stunted, wasted, and underweight growth significantly decreases by $4.1 \%, 5.5 \%$, and $6.2 \%$, respectively. A previous study highlights similar results that FCS is significantly associated with child health outcomes (Saaka and Osman, 2013).

Male children were more likely to have far worse health outcomes perhaps due to unobserved biological factors. Significant results on maternal age indicate that children born to women who are older are more likely to be healthier. As the mother gets older, she accrues the freedom of selfgovernance, better child-care practices and can effectively utilize resources. 
Maternal education, which was used as a proxy for the women's nutritional knowledge provided mixed results, in line with existing literature. Being educated does not necessarily translate into health and nutrition knowledge. Therefore, this study calls for further research on the relationship between the knowledge of nutritional requirements and child health outcomes. Similarly, working status of the mother has provided mixed results. On the brighter side, working mothers can provide basic requirements essential for the growth and development of children. However, in their absence, the children are often left under the care of relatives or baby care centers, with caregivers who may be ignorant, arrogant, and neglect the child's nutritional needs.

Furthermore, results show that households with improved sources of drinking water and sanitation facilities are less likely to have children who suffer from undernutrition. Contrary to theoretical expectations, children born into households with improved sanitation facilities were significantly more likely to suffer from wasted growth (Table 8). This is corroborated with the mixed findings on education and working status of the mother. 
Table 6. Households that lacked food/enough money to purchase food and child health outcomes

\begin{tabular}{|c|c|c|c|}
\hline \multirow{2}{*}{ Variable } & Stunted & Wasted & Underweight \\
\hline & OR (z statistic) & OR (z statistic) & OR (z statistic) \\
\hline \multicolumn{4}{|l|}{ Second-stage regression (Structural equation) } \\
\hline $\begin{array}{l}\text { Households that lacked food/enough money to } \\
\text { purchase food ( } 1=\text { Yes })\end{array}$ & $1.940(3.80)^{*}$ & $2.991(3.10)^{* *}$ & $2.890(4.64)^{* * *}$ \\
\hline $\begin{array}{l}\text { Residual households that lacked food/enough } \\
\text { money to purchase food }\end{array}$ & $0.778(-3.02)^{*}$ & $0.661(-2.41)^{*}$ & $0.670(-3.61)^{* * *}$ \\
\hline Child's gender (1=Male) & $1.455(7.72)^{* * *}$ & $1.329(2.90)^{* * *}$ & $1.283(3.92)^{* * *}$ \\
\hline Child's age (months) & $1.011(7.23) * * *$ & $0.995(-1.65)$ & $1.013(6.80)^{* * *}$ \\
\hline Maternal age (years) & $0.991(-2.35)^{*}$ & $1.006(0.82)$ & $0.999(-0.07)$ \\
\hline \multicolumn{4}{|l|}{ Maternal education } \\
\hline (1=Primary) & $1.118(1.72)$ & $0.297(-10.37)^{* * *}$ & $0.579(-7.08)^{* * *}$ \\
\hline (1=Secondary) & $0.727(-3.54) * * *$ & $0.313(-6.60)^{* * *}$ & $0.362(-8.27)^{* * *}$ \\
\hline$(1=$ Tertiary $)$ & $0.642(-2.98) * *$ & $0.124(-4.76) * * *$ & $0.307(-5.11)^{* * *}$ \\
\hline Mother's working status ( $1=$ Yes $)$ & $1.169(2.95)^{* *}$ & $0.806(-2.02)^{*}$ & $1.015(0.22)$ \\
\hline Area of residence (1=Rural) & $1.095(1.56)$ & $1.064(0.52)$ & $1.131(1.56)$ \\
\hline Source of drinking water ( $1=$ Improved $)$ & $0.827(-3.68) * * *$ & $1.151(1.36)$ & $0.877(-1.96) *$ \\
\hline Type of sanitation facility ( $1=$ Improved) & $0.848(-2.88) * * *$ & $1.120(0.94)$ & $0.857(-1.99)^{*}$ \\
\hline Constant & $0.229(-9.07) * * *$ & $0.061(-8.81)^{* * *}$ & $0.100(-10.92)^{*}$ \\
\hline \multicolumn{4}{|c|}{ First stage logistic regression (Reduced form equation) } \\
\hline \multicolumn{4}{|c|}{ Wealth index, OR ( $\mathrm{p}$ value) } \\
\hline (1=Middle) & $0.651(<0.001)^{* * *}$ & & \\
\hline (1=Rich) & $0.313(<0.001) * * *$ & & \\
\hline Ownership of land (1=Yes), OR ( $\mathrm{p}$ value) & $0.615(<0.001)^{* * *}$ & & \\
\hline Number of observations & 9,007 & & \\
\hline LR test statistic (p value) & $321.47(<0.001)^{* * *}$ & $244.40(<0.001)^{* * *}$ & $359.37(<0.001)^{* * *}$ \\
\hline Pseudo $\mathbf{R}^{2}$ & 0.031 & 0.067 & 0.051 \\
\hline
\end{tabular}

Source: Author's computation from KDHS (2014)

Notes: * for $\mathrm{p}<0.05, * *$ for $\mathrm{p}<0.01, * * *$ for $\mathrm{p}<0.001$ 
Table 7. Reduced CSI and child health outcomes

\begin{tabular}{|c|c|c|c|c|}
\hline \multirow{3}{*}{ Variable } & \multicolumn{3}{|c|}{ 2SRI-IV } & \multirow{2}{*}{ Wasted growth } \\
\hline & Stunted & Wasted & Underweight & \\
\hline & OR (z statistic) & OR (z statistic) & OR (z statistic) & OR (z statistic) \\
\hline \multicolumn{5}{|c|}{ Second-stage regression (Structural equation) } \\
\hline Reduced CSI & $1.076(2.68)^{* *}$ & $1.016(0.29)$ & $1.084(2.23)^{*}$ & $1.010(1.91)$ \\
\hline Residual Reduced CSI & $0.932(-2.54)^{*}$ & $0.994(-0.12)$ & $0.931(-1.96) *$ & \\
\hline Child's gender (1=Male) & $1.457(4.81) * * *$ & $1.276(1.66)$ & $1.144(1.38)$ & $1.281(1.73)$ \\
\hline Child's age (months) & $1.013(5.46)^{* * *}$ & $0.998(-0.45)$ & $1.015(5.22)^{* * *}$ & $0.998(-0.44)$ \\
\hline Maternal age (years) & $0.991(-1.53)$ & $0.995(-0.39)$ & $0.993(-0.89)$ & $0.996(-0.38)$ \\
\hline \multicolumn{5}{|l|}{ Maternal education } \\
\hline$(1=$ Primary $)$ & $1.293(2.28)^{*}$ & $0.229(-6.95)^{* * *}$ & $0.526(-4.76)^{* * *}$ & $0.226(-8.84)^{* * *}$ \\
\hline (1=Secondary) & $0.798(-1.17)$ & $0.272(-3.48)^{* * *}$ & $0.414(-3.49) * * *$ & $0.264(-4.93)^{* * *}$ \\
\hline (1=Tertiary) & $0.976(-0.06)$ & $0.109(-2.05) *$ & $0.523(-1.22)$ & $0.105(-2.20)^{*}$ \\
\hline Mother's working status ( $1=$ Yes $)$ & $1.243(2.52)^{*}$ & $0.970(-0.18)$ & $1.172(1.46)$ & $0.965(-0.23)$ \\
\hline Area of residence $(1=$ Rural $)$ & $1.091(0.91)$ & $1.145(0.73)$ & $1.278(1.98)^{*}$ & $1.145(0.73)$ \\
\hline Source of drinking water (1=Improved) & $0.901(-1.27)$ & $1.044(0.28)$ & $0.915(-0.88)$ & $1.039(0.26)$ \\
\hline Type of sanitation facility (1=Improved) & $0.741(-3.26)^{* * *}$ & $1.151(0.80)$ & $0.817(-1.71)$ & $1.150(0.79)$ \\
\hline Constant & $0.066(-4.68)^{* * *}$ & $0.107(-1.95)$ & $0.039(-4.28)^{* * *}$ & $0.121(-5.17)^{* * *}$ \\
\hline \multicolumn{5}{|c|}{ First stage linear regression (Reduced form equation) } \\
\hline \multicolumn{5}{|l|}{ Wealth index, coefficient ( $\mathrm{p}$ value) } \\
\hline (1=Middle) & $-2.035(0.002)^{* *}$ & & & \\
\hline (1=Rich) & $-3.765(<0.001) * * *$ & & & \\
\hline $\begin{array}{l}\text { Number of children in the household } \\
\text { under-five years, coefficient ( } p \text { value) }\end{array}$ & $0.916(0.001)^{* * *}$ & & & \\
\hline Number of observations & 3,380 & & & \\
\hline LR test statistic ( $p$ value) & $138.62(<0.001) * * *$ & $117.51(<0.001)^{* * *}$ & $165.82(<0.001)^{* * *}$ & $117.49(<0.001) * * *$ \\
\hline Pseudo $\mathbf{R}^{2}$ & 0.033 & 0.072 & 0.054 & 0.072 \\
\hline
\end{tabular}

Source: Author's computation from KDHS (2014)

Notes: * for $\mathrm{p}<0.05, * *$ for $\mathrm{p}<0.01, * * *$ for $\mathrm{p}<0.001$ 
Table 8. Food Consumption Score and child health outcomes

\begin{tabular}{|c|c|c|c|}
\hline \multirow{2}{*}{ Variable } & Stunted & Wasted & Underweight \\
\hline & OR (z statistic) & OR (z statistic) & OR (z statistic) \\
\hline \multicolumn{4}{|l|}{ Second-stage regression (Structural equation) } \\
\hline FCS & $0.959(-5.95)^{* * *}$ & $0.945(-3.77)^{* * *}$ & $0.938(-6.65)^{* * *}$ \\
\hline Residual FCS & $1.038(5.29) * * *$ & $1.053(3.40) * * *$ & $1.061(6.08)^{* * *}$ \\
\hline Child's gender ( $1=$ Male $)$ & $1.462(7.81)^{* * *}$ & $1.337(2.97) * *$ & $1.292(4.02)^{* * *}$ \\
\hline Child's age (months) & $1.010(7.01)^{* * *}$ & $0.995(-1.83)$ & $1.013(6.59)^{* * *}$ \\
\hline Maternal age (years) & $0.992(-2.21)^{*}$ & $1.007(0.95)$ & $1.001(0.10)$ \\
\hline \multicolumn{4}{|l|}{ Maternal education } \\
\hline (1=Primary) & $1.161(2.27)^{*}$ & $0.313(-9.79)^{* * *}$ & $0.617(-6.16)^{* * *}$ \\
\hline (1=Secondary) & $0.801(-2.39)^{*}$ & $0.353(-5.69)^{* * *}$ & $0.423(-6.81)^{* * *}$ \\
\hline (1=Tertiary) & $0.905(-0.59)$ & $0.194(-3.46)^{* * *}$ & $0.533(-2.45)^{*}$ \\
\hline Mother's working status $(1=$ Yes $)$ & $1.169(2.95)^{* *}$ & $0.815(-1.91)$ & $1.025(0.36)$ \\
\hline Area of residence (1=Rural) & $0.983(-0.28)$ & $0.909(-0.74)$ & $0.957(-0.52)$ \\
\hline Source of drinking water (1=Improved) & $0.882(-2.37)^{*}$ & $1.218(1.88)$ & $0.952(-0.71)$ \\
\hline Type of sanitation facility (1=Improved) & $0.985(-0.23)$ & $1.324(2.07)^{*}$ & $1.061(0.67)$ \\
\hline Constant & $2.937(2.71)^{* *}$ & $2.245(0.94)$ & $5.389(3.05)^{* *}$ \\
\hline \multicolumn{4}{|c|}{ First stage linear regression (Reduced form equation) } \\
\hline \multicolumn{4}{|l|}{ Wealth index, coefficient ( $\mathrm{p}$ value) } \\
\hline (1=Middle) & $4.628(<0.001) * * *$ & & \\
\hline (1=Rich) & $10.479(<0.001) * * *$ & & \\
\hline Ownership of land (1=Yes), coefficient ( $p$ value) & $3.467(<0.001) * * *$ & & \\
\hline Number of observations & 9,018 & & \\
\hline LR test statistic (p value) & $347.10(<0.001) * * *$ & $250.50(<0.001)^{* * *}$ & $385.49(<0.001) * * *$ \\
\hline Pseudo $\mathbf{R}^{2}$ & 0.033 & 0.069 & 0.055 \\
\hline
\end{tabular}

Source: Author's computation from KDHS (2014)

Notes: * for $\mathrm{p}<0.05, * *$ for $\mathrm{p}<0.01, * * *$ for $\mathrm{p}<0.001$ 


\section{Conclusion}

One of the key pillars under Kenya's Big Four Agenda is to ensure that everyone, at all times, has physical, economic, and social access to adequate and nutritious food which is essential in improving health. Although more than half of the population in Kenya is food secure, $37 \%$ of those who lack minimum access to adequate and nutritious food is still a call for concern. This study sought to estimate the effect of household food security status on child health outcomes in Kenya using the KDHS (2014) dataset. Due to potential endogeneity, a structural health production function was estimated using the two-stage residual inclusion (2SRI) technique. We used three measures of household food security status to determine its effect on undernutrition in children. Our key findings showed a positive and significant association between the households that lacked food/enough money to purchase food and the FCS with child health outcomes (stunting, wasting, and underweight growth in children under-five years). Although the Reduced CSI was positively associated with stunting and underweight in children, there was no significant association with wasted growth. Our findings, therefore, suggest that worsening food security status compromises child health in Kenya.

Our results are also somewhat consistent with existing literature that shows that depending on the measurement instrument, the effect of household food security status on child nutritional outcomes may differ. Contrary to theoretical expectations, children born into households with improved sanitation facilities were significantly more likely to suffer from wasted growth (Table 8). This is corroborated with the mixed findings on education and working status of the mother. There is also likely to be an undetected adverse effect on child health outcomes from unfavorable maternity leave policies and working conditions on career and working mothers. With mothers working more hours and leaving their young children with less educated caretakers, it is likely that our models capture the impact of caretakers' characteristics on child health outcomes. This calls for workplace policies that support motherhood and career development for women without compromising future economic growth and development.

Informed by evidence, one of the major recommendations aimed at promoting child health outcomes in Kenya is to implement social assistance programs to enable households to cushion themselves from food insecurity. Specifically, the government should implement food aid interventions such as school feeding programs especially in areas prone to food insecurity. Unsafe water and unimproved sanitation facilities result in ingestion of fecal pathogens that have not been properly disposed of. As such, nutrition intervention programs should be integrated with WASH interventions to facilitate the assimilation of essential nutrients in food. Capacity-building programs should also be implemented to provide a platform for women, 
regardless of their educational background to acquire knowledge on health, nutrition, and better child-care practices.

One of the major limitations of this study was that it was based on crosssectional data which made it impossible to model and analyze the stability of household food security status on child health outcomes. Therefore, there is a need for further research using experimental methodologies like Randomized Controlled Trials (RCTs) to determine the causal impact between food/nutrition security and child health outcomes. In the absence of selection bias, RCTs can provide an unbiased and true estimate of the impact measured by the difference in desired outcomes between the treatment and control groups.

\section{References:}

1. Action Against Hunger. (2017). WASH' Nutrition: A Practical Guidebook on Increasing Nutritional Impact Through Integration of WASH and Nutrition Programmes. Paris, France: ACF International.

2. Bascle, G. (2008). Controlling for Endogeneity with Instrumental Variables in Strategic Management Research. Strategic Organization, 6(3), 285-327.

3. Bloom, D. E., \& Canning, D. (2008). Population Health and Economic Growth (Working Paper No. 24). Washington, DC: World Bank.

4. Blössner, M., \& de Onis, M. (2005). Malnutrition: Quantifying the Health Impact at National and Local Levels (WHO, Environmental Burden of Disease Series, No. 12). Geneva: WHO.

5. Bound, J., Jaeger, D. A., \& Baker, R. M. (1995). Problems with Instrumental Variables Estimation When the Correlation Between the Instruments and the Endogenous Explanatory Variable is Weak. Journal of the American Statistical Association, 90(430), 443-450.

6. Food and Agriculture Organization. (2009). Declaration of the World Summit on Food Security. World Summit on Food Security (pp. 1-7). Rome: FAO.

7. Hackett, M., Melgar-Quiñonez, H., \& Álvarez, M. C. (2009). Household Food Insecurity Associated with Stunting and Underweight among Preschool Children in Antioquia, Colombia. Revista Panamericana de Salud Pública, 25 (6), 506-510.

8. Huffman, W. E. (2010). Household Production Theory and Models (Working Paper No. 10019). Ames, Iowa: Iowa State University.

9. Jemal, Z., Hassen, K., \& Wakayo, T. (2016). Household Food Insecurity and its Association with Nutritional Status among Preschool Children in Gambella Town, Western Ethiopia. Journal of Nutrition \& Food Sciences, 6 (6), 566. doi: 10.4172/2155-9600.1000566. 
10. Kac, G., Schlüssel, M. M., Pérez-Escamilla, R., Velásquez-Melendez, G., \& Moura da Silva, A. A. (2012). Household Food Insecurity Is Not Associated with BMI for Age or Weight for Height among Brazilian Children Aged 0-60 Months. Plos One, 7 (9), e45747. doi:10.1371/journal.pone.0045747.

11. Kenya National Bureau of Statistics. (2018). Basic Report on Wellbeing in Kenya: Based on the 2015/16 Kenya Integrated Household Budget Survey (KIHBS). Nairobi: KNBS.

12. Kenya National Bureau of Statistics, Ministry of Health/Kenya, National AIDS Control Council/Kenya, Kenya Medical Research Institute, National Council for Population and Development/Kenya and ICF International. (2015). Kenya Demographic and Health Survey 2014. Rockville, MD, USA: KNBS and ICF International.

13. Maxwell, D., \& Caldwell, R. (2008). The Coping Strategies Index: Field Methods Manual, Second Edition. Cooperative for Assistance and Relief Everywhere, Inc. (CARE).

14. Meerman, J., \& Aphane, J. (2012). Impact of High Food Prices on Nutrition. FAO Expert Consultation on Policy Responses to High and Volatile Food Prices. FAO.

15. Mutisya, M., Kandala, N.-b., Ngware, M. W., \& Kabiru, C. W. (2015). Household food (in)security and nutritional status of urban poor children aged 6 to 23 months in Kenya. BMC Public Health, 15 (1), 1052. doi: 10.1186/s12889-015-2403-0.

16. Osei, A., Pandey, P., Spiro, D., Nielson, J., Shrestha, R., Talukder, Z., . . . Haselow, N. (2010). Household Food Insecurity and Nutritional Status of Children Aged 6 to 23 Months in Kailali District of Nepal. Food and Nutrition Bulletin, 31 (4), 483-494.

17. Rose, D., \& Bodor, J. N. (2006). Household Food Insecurity and Overweight Status in Young School Children: Results From the Early Childhood Longitudinal Study. Pediatrics, 117 (2), 464-473. doi:10.1542/peds.2005-0582.

18. Saaka, M., \& Osman, S. M. (2013). Does Household Food Insecurity Affect the Nutritional Status of Preschool Children Aged 6-36 Months? International Journal of Population Research, 2013, 304169. doi: 10.1155/2013/304169.

19. Saha, K. K., Frongillo, E. A., Alam, D. S., Arifeen, S. E., Persson, L. A., \& Rasmussen, K. M. (2009). Household Food Security is Associated with Growth of Infants and Young Children in Rural Bangladesh. Public Health Nutrition, 12 (9), 1556-1562. doi:10.1017/S1368980009004765. 
20. Shea, J. (1997). Instrument Relevance in Multivariate Linear Models: A Simple Measure. The Review of Economics and Statistics, 79(2), 348-352.

21. Singh, A., Singh, A., \& Ram, F. (2014). Household Food Insecurity and Nutritional Status of Children and Women in Nepal. Food and Nutrition Bulletin, 35 (1), 3-11.

22. Stock, J. H., Wright, J. H., \& Yogo, M. (2002). A Survey of Weak Instruments and Weak Identification in Generalized Method of Moments. Journal of Business \& Economic Statistics, 20(4), 518-529.

23. Terza, J. V., Basu, A., \& Rathouz, P. J. (2008). Two-Stage Residual Inclusion Estimation: Addressing Endogeneity in Health Econometric Modeling. Journal of Health Economics, 27(3), 531-543.

24. The Economist Intelligence Unit. (2017). Global Food Security Index 2017: Measuring Food Security and The Impact of Resource Risks. New York: The Economist Intelligence Unit Limited.

25. The Economist Intelligence Unit. (2018). Global Food Security Index 2018: Building Resilience in the Face of Rising Food-Security Risks. New York: The Economist Intelligence Unit Limited.

26. United Nations. (2015). Transforming Our World: The 2030 Agenda for Sustainable Development. Sustainable Development Knowledge Platform. Online. (https:// sustainabledevelopment.un.org/post2015/transformingourworld)

27. United Nations Development Programme. (2019). Goal 3: Good Health and Well-Being. Retrieved from http://www.undp.org/content/undp/en/home/sustainabledevelopment-goals/goal-3-good-health-and-well-being.html Accessed on $30 / 4 / 19$

28. WFP (World Food Programme), VAM (Vulnerability Analysis and Mapping). (2006). Food Consumption Analysis: Calculation and Use of the Food Consumption Score in Food Security Analysis. Rome: World Food Programme, Vulnerability Analysis and Mapping Branch (ODAV).

29. WHO (World Health Organization). (2006). WHO Child Growth Standards: Length/height-for-age, Weight-for-age, Weight-for-length, Weight-for-height and Body Mass Index-for-age: Methods and Development. Geneva: WHO.

30. World Bank. (1993). World Development Report 1993: Investing in Health. New York: Oxford University Press, Inc. 